\title{
Effect of Doxycycline in Autologous Serum to Improvement Clinical Manifestation of Acne Vulgaris
}

\author{
Muhlis Yunus ${ }^{1}$, Anis Irawan Anwar ${ }^{1}$, Siswanto Wahab ${ }^{1}$, Farida Tabri ${ }^{1}$, Ilhamjaya Patellongi ${ }^{2}$, \\ Johanes Widodo 3 \\ ${ }^{1}$ Department of Dermatology and Venereology, Medical Faculty, Hasanuddin University, Makassar, Indonesia \\ ${ }^{2}$ Department of Biostatistic, Faculty of Public Health, Hasanuddin University, Makassar, Indonesia \\ ${ }^{3}$ Department of Dermatology and Venereology, Medical Faculty, Gadjah Mada University, Jogjakarta, Indonesia
}

Email address:

dr.muhlis@gmail.com (M. Yunus)

\section{To cite this article:}

Muhlis Yunus, Anis Irawan Anwar, Siswanto Wahab, Farida Tabri, Ilhamjaya Patellongi, Johanes Widodo. Effect of Doxycycline in Autologous Serum to Improvement Clinical Manifestation of Acne Vulgaris. American Journal of Clinical and Experimental Medicine. Vol. 5, No. 2, 2017, pp. 30-35. doi: 10.11648/j.ajcem.20170502.11

Received: January 25, 2017; Accepted: February 10, 2017; Published: March 1, 2017

\begin{abstract}
Acne vulgaris (AV) is an inflammatory disease of skin on the follicle polisebasea frequent and considered dermatological problems the most common worldwide. This study investigates the relationship topical therapy using autologous serum containing doxycycline in women with moderate to severe acne vulgaris. Research conducted in Makassar, South Sulawesi, as many as 13 samples that met the inclusion criteria. Clinical examination in patients with moderate acne vulgaris to severe and based on clinical manifestations, level of sebum measured before, and levels of estradiol. All patients given the autologous serum after being given doxycycline $200 \mathrm{mg}$ single dose oral which will be applied every night. Furthermore monitored on day to 14 and day to 28 . The data were processed using paired Wilcoxon test. Results improvement in clinical manifestations and levels of sebum on days 14 and 28 compared to day 1 was significantly $(p<0.05)$. There were improvements in clinical manifestations and decrease sebum levels in patients with moderate to severe AV after application of autologous serum containing doxycycline.
\end{abstract}

Keywords: Acne Vulgaris, Autologous Serum, Doxycycline, Estradiol

\section{Introduction}

Acne vulgaris (AV) is an inflammatory disease of the follicle polisebasea skin that often occurs and is considered dermatological problems. [1] The pathogenesis of acne vulgaris consists of four which is the key the occurrence of acne vulgaris include excessive sebum production, abnormal keratinization (hiperkeratinisasi), colonization of Propionibacterium acnes (P. acnes), release of inflammatory mediators. [2] The use of systemic antibiotics recommended for the treatment of moderate to severe acne vulgaris is doxycycline and minocycline. The peak plasma concentration reported 2 hours after an oral dose of $200 \mathrm{mg}$ in 24 hours. [3]

Topical treatment for acne vulgaris is retinoid (adapalene, isotretinoin, tretinoin and others, antibiotics (clindamycin, erythromycin) and other topical. Failure of therapy with standard treatments commonly found in patients with acne vulgaris. [3, 4] Estrogen is known estron (E1), estradiol (E2), and estriol (E3), estradiol is the most active. Estradiol levels menstrual phase is $40-200 \mathrm{pg} / \mathrm{mL}$. The components in the blood including plasma consists of water, proteins, hormones that are part of the serum. Serum taken from patient's blood called autologous serum. [5, 6] Examination to determine levels estradiol using by Enzyme-Linked immunosorbent Assay (ELISA). [7] There are conditions can not given oral therapy administered or have contraindicated to use systemic drugs for treatment of acne vulgaris, for example in pregnant, use derivatives of vitamin A and others. Until now there has been no preparation of topical doxycycline.

To determine the content of doxycycline in autologous serum can improvement with acne vulgaris patients, serum estradiol levels taken at a low in the blood when the menstrual period. $[8,9]$ We want to know correlation topical 
therapy using autologous serum contains doxycycline in women to improvement with moderate to severe acne vulgaris patients.

\section{Materials and Methods}

\subsection{Location and Research Time}

The study was conducted at the Polyclinic Dermatovenerelogy Wahiddin Sudirohusodo General Hospital and Networking begin July - August 2016.

\subsection{Design Study}

This is clinical trial study design with prospectivepre and post treatment method. Criteria inclusion are women with moderate to severe acne vulgaris, 15-30 years, menstrual cycles every month, have not history topical treatment two weeks, did not receive antibiotics or corticosteroid systemic one month, did not receive contraceptive 3 month. exclusion criteria are pregnant patient, have receive oral contraceptive, have not refrigerator.

\subsection{Procedure}

The patient given doxycycline $200 \mathrm{mg} 2$ hours before taking a blood sample from median cubital vein of $40 \mathrm{cc}$ with antiseptic manner using vacutainer $25 \mathrm{G}$ needle inserted into the tube, and the blood will be centrifuges for 10-15 minutes at $3000 \mathrm{rpm}$, in which the serum will be separated above, and will be stored in refrigerator with the temperature $-20^{\circ} \mathrm{C}$. Patients applied serum topically every night, sebum level and clinical manifestation will be meassured again on day 14 and day 28 after the start of research.

\subsection{Analysis of Data}

Data in this study will be processed using statistic softwere, SPSS Version 21.0. All analysis results will be presented in tables with explanation. Statistical test used was Wilcoxon test. The hypothesis will be accepted if then $p$ value $<0.05$.

\section{Results}

Thirteen patients who meet the criteria of the study sample, observation day-1 day-14 and day-28 of the number of comedones, papules, pustules and sebum. Only estradiol levels checked on day-1 to ensure the samples are in the menstrual phase. The results of analysis the descriptive each variable research can be seen in table 1 . It can be seen that the highest levels of estradiol $200.5 \mathrm{pg} / \mathrm{mL}$ with a mean of 70.39; lower than preovulasi. The average value number of comedones, papules, pustules and sebum decreases after 14 days and decreased again after 28 days.

Table 1. Descriptive Statistics.

\begin{tabular}{lllllll}
\hline Variable & $\mathbf{n}$ & Observation & Minimum & Maksimum & Mean & SD \\
\hline Estradiol Level & 13 & & 20,8 & 200,5 & 70,39 & 43,86 \\
Total Comedo & 13 & & 18 & 43 & 30,0 & 7,9 \\
Total Papule & 13 & Day-1 & 3 & 17 & 8,9 & 4,4 \\
Total Pustule & 13 & & 4 & 16 & 7,7 & 3,6 \\
Level Sebum & 13 & & 79 & 220 & 127,3 & 39,2 \\
Total Comedo & 13 & & 12 & 36 & 21,5 & 7,9 \\
Total Papule & 13 & Day-14 & 3 & 11 & 6,9 & 2,1 \\
Total Pustule & 13 & & 1 & 11 & 4,9 & 2,8 \\
Level Sebum & 13 & & 59 & 143 & 92,0 & 27,7 \\
Total Comedo & 13 & & 5 & 25 & 12,2 & 5,7 \\
Total Papule & 13 & & 1 & 7 & 4,2 & 1,7 \\
Total Pustule & 13 & Day-28 & 0 & 3 & 140 \\
Level Sebum & 13 & & 48 & 1,3 & 2,0 \\
\hline
\end{tabular}

Variable Observations n Mean SD Minimum Maximum Levels of Estradiol

Table 2. Differences in the number of comedones, papules, pustules and sebum is based on the observation.

\begin{tabular}{|c|c|c|c|}
\hline \multirow{3}{*}{ Variable } & \multicolumn{3}{|l|}{ Observation } \\
\hline & Day-1 & Day-14 & Day-28 \\
\hline & Mean (SD) & Mean (SD) & Mean (SD) \\
\hline Total Comedo & $30,0(7,9)^{\mathrm{c}}$ & $21,5(7,9)^{b}$ & $12,2(5,7)^{\mathrm{a}}$ \\
\hline Total Papule & $8,9(4,4)^{\mathrm{c}}$ & $6,9(2,1)^{\mathrm{b}}$ & $4,2(2,0)^{\mathrm{a}}$ \\
\hline Total Pustule & $7,7(3,6)^{\mathrm{c}}$ & $4,9(2,8)^{\mathrm{b}}$ & $1,7(1,3)^{\mathrm{a}}$ \\
\hline Level Sebum & $127,3(39,2)^{\mathrm{c}}$ & $92,0(27,7)^{\mathrm{b}}$ & $73,0(23,5)^{a}$ \\
\hline
\end{tabular}

Superscript berebeda on the same line Wilcoxon test results showed significant $(\mathrm{p}<0.05)$; when same means was not significant $(\mathrm{p}>0.05)$ 
Table 2 can be seen the results of Wilcoxon test results showed comedones, papules, pustules and sebum between observation day-1, day-14 and day-28. Shows that occur to a significant reduction $(p<0.05)$ the average number of blackheads on day 14 and remained decreased at day- 28 . Similary the number of papules, pustules and sebum.
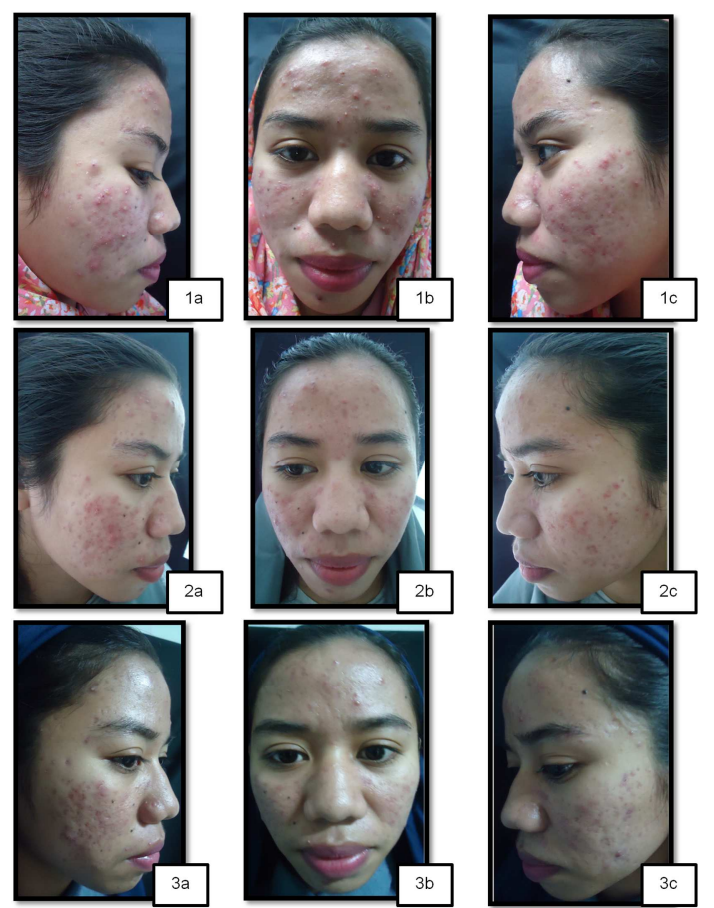

Figure 1. Improvement of clinical manifestation on day $1(1 a, b, c)$, day 14 $(2 a, b, c)$, and $28(3 a, b, c)$.
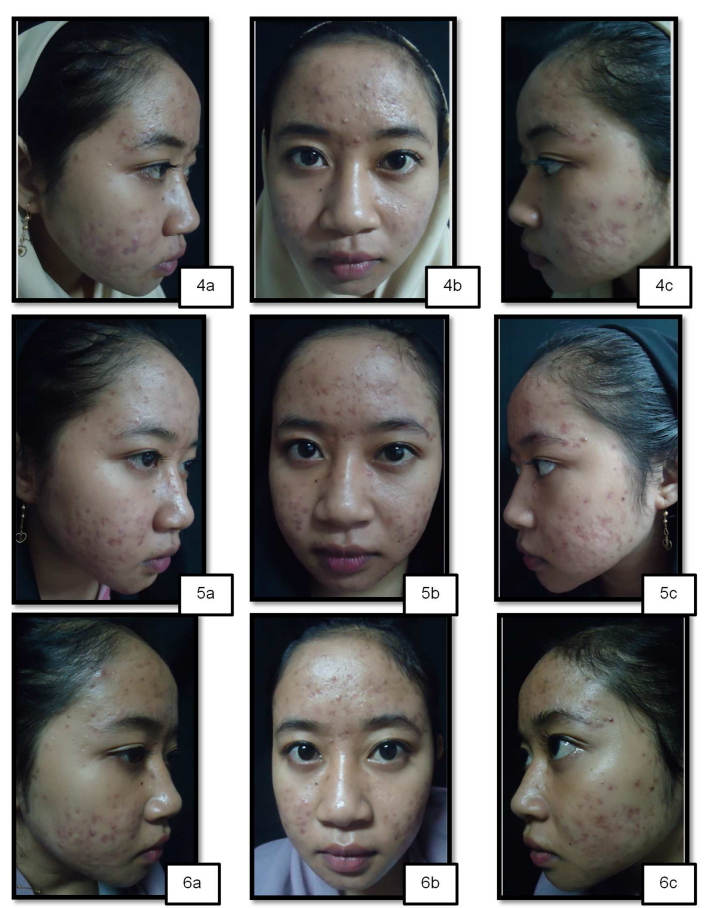

Figure 2. Improvement of clinical manifestation on day $1(4 a, b, c)$, day 14 $(5 a, b, c)$, and $28(6 a, b, c)$.
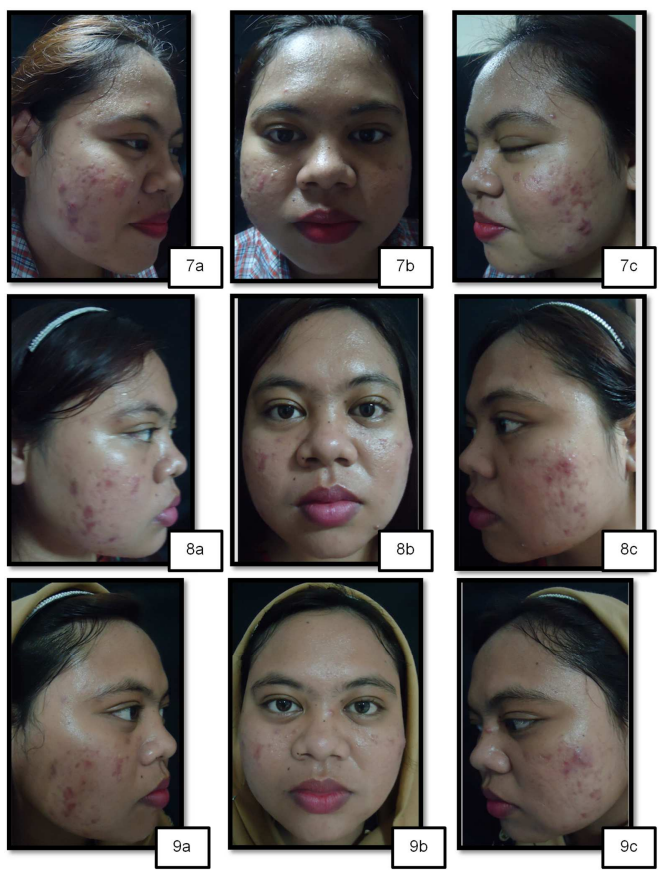

Figure 3. Improvement of clinical manifestation on day 1 (7a, b, c), day 14 $(8 a, b, c)$, and $28(9 a, b, c)$.

Table 3. Percentage decrease in the number of comedones, papules, pustules and sebum after day 14 and day 28 post-application of autologous serum containing doxycycline.

\begin{tabular}{llllll}
\hline \multirow{2}{*}{ Variable } & D1 - D14 & \multicolumn{3}{l}{ D1 - D28 } \\
\cline { 2 - 5 } & Mean (SD) & p $^{*}$ & Mean (SD) & p* \\
\hline Decreased Comedo (\%) & $29,2(12,0)$ & $<0,001$ & $60,2(11,7)$ & $<0,001$ \\
Decreased Papul (\%) & $20,1(20,1)$ & $<0,001$ & $54,4(15,0)$ & $<0.001$ \\
Decreased Pustul (\%) & $37,4(26,8)$ & $<0,001$ & $78,9(15,4)$ & $<0,001$ \\
Decreased Level Sebum (\%) & $25,4(17,0)$ & $<0,001$ & $41,2(12,5)$ & $<0,001$ \\
\hline
\end{tabular}

${ }^{*}$ Wilcoxon test; H1 = day-1, H14 = day 14, and H28 = day-28

Table 3 it can be seen that the decline in the number of blackheads with the average percentage of $29.2 \%$ on day 14 and the average percentage of $60.2 \%$ on the day- 28 after the application of autologous serum containing doxycycline. Neither the number of papules, pustules and sebum; respectively decreased $20.1 \%$ on day 14 and $54.4 \%$ at day 28 for papules; $37.4 \%$ on day 14 and $78.9 \%$ at day 28 for pustules; and $25.4 \%$ on day 14 and $41.2 \%$ at day 28 for the sebum.

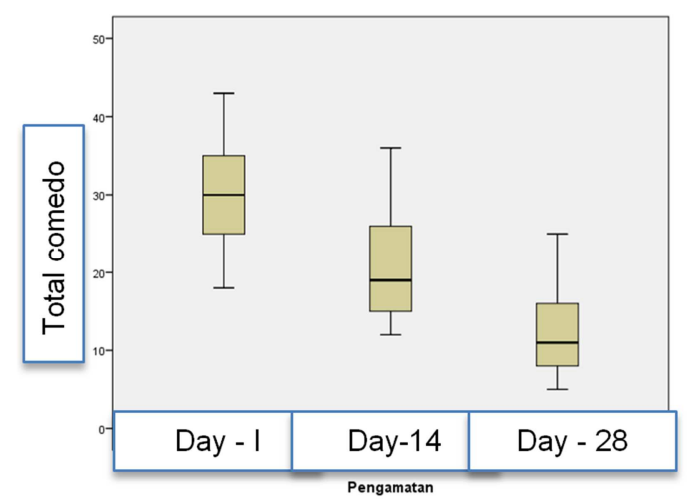

Figure 4. Shows the reduction in the number of blackheads on day 14 and declined again on day 28. 


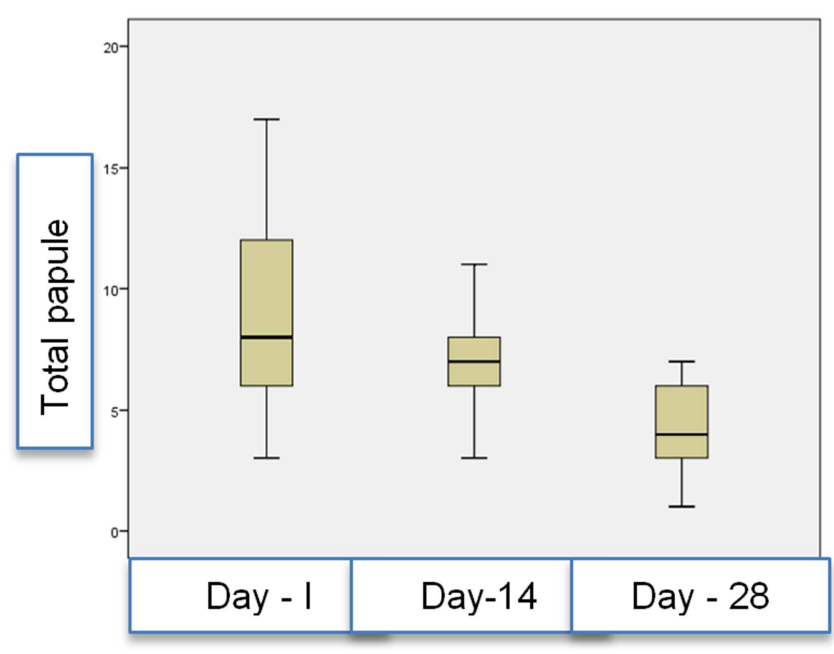

Figure 5. Shows the decrease in the number of papules on day 14 and declined again on day 28.

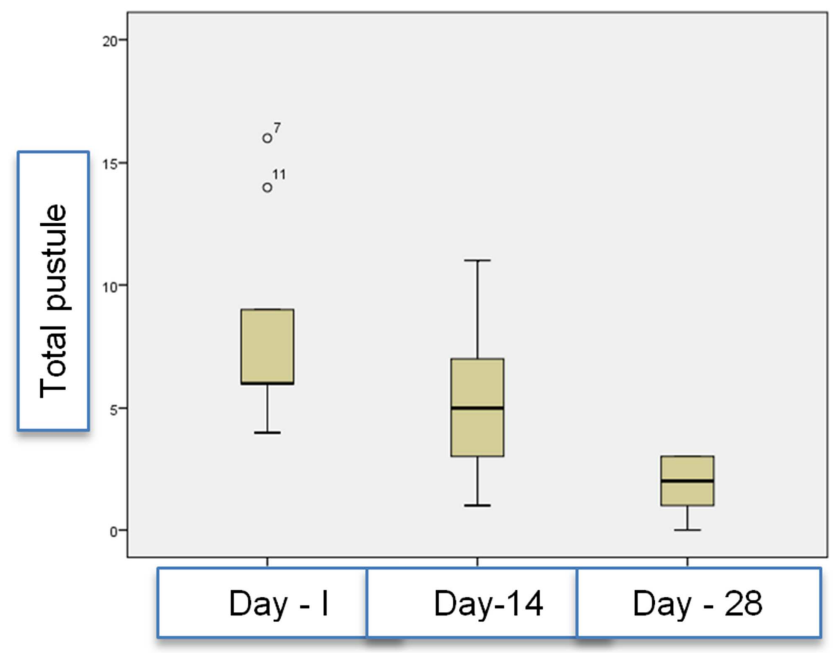

Figure 6. Shows a decrease in the number of pustules on days-14 and decreased again on day 28.

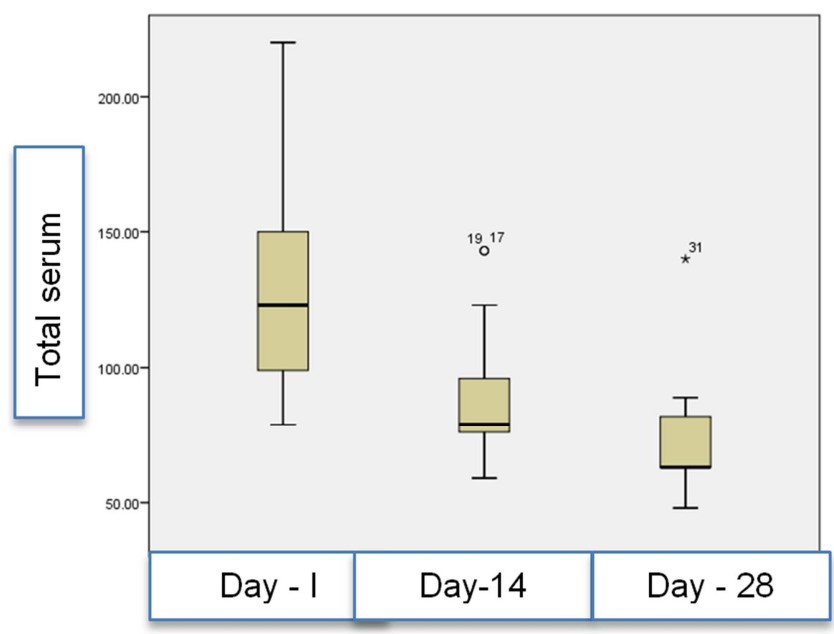

Figure 7. Shows a decrease sebum on day 14 and declined again on day 28.

\section{Discussion}

In acne vulgaris hormonal influences play an important role. [10] Keratinocytes in normal follicle cells are always present in a single lumen issued. In patients with acne vulgaris, keratinocyte hyperproliferation buildup resulted in desquamation of corneocytes abnormal sebaceous follicles along with lipid and monofilament, phenomenon this which produces comedogenesis. P.acne are anaerobic, gramnegative pathogens colonizing the sebaceous follicles. [11] Generally on the skin area where there are many sebaceous follicles as sebum production in large quantities, lipids that many anaerobic environments are optimal for $P$ acnes. Inflammatory process starts when $P$ acnes on detection by the system, immune then the effect of severe inflammation be a spark discharge factors chemostatic such as lymphocytes, and macrophages neutrofil. [7] The factors that cause follicle damage, rupture and the entry of bacterial lipid acid, and contained in surrounding dermis. [12] This process will continue and lead to the formation of inflammatory lesions such as pustules, nodules, cysts and papules. [13, 14] Based on this study, the number of lesions on day 14 and day 28 is reduced significantly when compared with the first day. Autologous serum application containing doxycycline shows the influence on the improvement of acne vulgaris lesions where we know that the pathogenesis of acne primarily on process sebum comedogenesis plays a role where production levels sebum were higher in patients with severe AV. [15] The colonization of $P$. acne is a bacteria gram positive commensal epidermis at the early stage development of inflammatory lesions of acne. [15, 16]

Frequently a topical antibiotic used for the treatment of acne is erythromycin, clindamycin, and tetracycline, but often resistent. In this study, blood sampling performed after administration of doxycycline $200 \mathrm{mg}$ a single dose so it is considered that the autologous serum containing doxycycline. Until now there has been no antibiotic topical doxycycline preparations. In this study, by administering serum acontaining doxycycline provides clinical improvement. Doxycycline is an analog of tetracycline, the antibiotic tetracycline main working mechanisms topical for the treatment of acne vulgaris by inhibiting inflammation caused by Propionibacterium acnes. [4, 17] Taking blood taken 2 hours after administration of doxycycline orally, it is based on the pharmacokinetics of doxycycline that reach peak concentration in plasma was reported two hours after administration of $200 \mathrm{mg}$ orally. [18] Doxycycline is one very effective oral antibiotics for acne inflammation that can be used as an acne treatment because it effectively inhibits the growth P.acne and has anti-inflammatory properties.

The use of oral antibiotics are generally indicated for inflammatory acne of moderate to severe, resistant to topical therapy earlier. Antibiotics Oral such as macrolides, tetracyclines (doxycycline, minocycline and limesiklin). The antibiotic is an agent that inhibits colonization $P$ acnes and synthesize inflammatory mediators of $P$ acnes tetracycline analogue to used often because it is effective and in expensive. Doxycycline and minocycline are preferred because the effect of lower gastrointestinal irritation and fat soluble, and penetration of the follicle polisebasea more 
efficiently. In addition to resistance $P$ acnes is lower than the reported class of macrolides. [16, 19, 20]

In this study, there is a significant reduction in the amount level of sebum

on day 14 and day 28 compared to day 1 after administration of autologous serum in which the $p$ value of $<0.05$. Sebum continuously be synthesized by the sebaceous glands and secretion to the surface of the skin through the pores of the hair follicles. The imbalance between the production of sebum secretion and capabilities will be micro comedo and can develop into inflammatory lesions. [13, 14, 21].

Androgen hormones stimulate increased production and secretion of sebum. Increased sebum production are directly related to the incidence of severity of acne lesions. [10] The hormone estrogen has a mechanism of opposition to androgens in sebaceous glands. In autologous serum one ingredient is a hormone estrogen. [12, 22] Estrogen is known estradiol, estrone, and estriol, estradiol is the most active. Oral contraceptives used for the treatment of acne vulgaris have contains estradiol, mechanism of action estradiol is decreases the activity of 5- $\alpha$ reductase, reduce the activity of sebaceous glands. High estradiol levels have been shown to be used for the treatment of acne vulgaris. [5, 6] To determine the content of doxycycline in autologous serum can improvement with acne vulgaris patients, serum estradiol levels taken at a low in the blood when the menstrual period, ELISA examination to determine levels estradiol. [7]

In this study, the menstrual estradiol (follicular phase) at levels get between 20.8 to $200.5 \mathrm{pg} / \mathrm{mL}$. The concentration of estradiol levels the lowest during the menstrual phase with a concentration in the serum of about 40 to $200 \mathrm{pg} / \mathrm{mL}$. While estradiol levels when preovulasi about 250 to $500 \mathrm{pg} / \mathrm{mL}$, it concluded that estrogen levels phase menstrual is lower than that of estradiol preovulasi phase. Low estrogen levels associated with the severity of acne. [5] Androgen hormones increase sebum production, so the sebum levels in patients with acne vulgaris higher than normal people. There is a correlation between sebum excretion and the severity of acne vulgaris because sebum levels in patients with severe acne vulgaris was found higher levels. Hormone estradiol may inhibit the production of sebum to reduce levels of sebum. The use of oral contraception for the treatment of acne vulgaris containing estradiol. [7, 14, 23] Study was conducted over day 28 , it is adjusted cyclically normal menstruation every month. The length of normal menstrual cycle is regarded as a classic menstrual cycle is day 28. [5, 24]

The main goal of treatment is control and treat acne lesions, prevent permanent scarring to a minimum, limiting the duration of the disruption and minimize morbidity. The treatment takes 3-6 weeks. [10] The study by Edison et al (2016) on the use of autologous serum containing high levels of estradiol treatment $\mathrm{AV}$ results occurred obtain clinical improvement and reduction in sebum after topical autologous serum. [25] Research conducted by Ilham 2011 using rats were divided into 5 groups were treated using eye drops autologous serum with various concentrations has an antibacterial effect because it contains some agents antibacterial include immunoglobulins, lysozyme and complement causes lysis of the bacteria and provide bacteriostatic, whereas anti-inflammatory effect of autologous serum based on the factors epiteliotrofik (epidermal growth factor, transforming growth factor $B$, fibrinogen and vitamin A) and neutrophilic (substance P, Nerve growth factor) which contain in the serum. [26] This study using autologous serum containing doxycycline in the menstrual phase to assess changes on patients with acne vulgaris, limitation of this study has no control.

\section{Conclusion}

Researchers concluded that there were clinical manifestations improvements in patients with moderatesevere acne vulgaris after the autologous serum application. There was a decreased sebum levels in patients with moderate-severe acne vulgaris after the autologous serum application. Researchers suggested that autologous serum contain doxycycline can be used as a therapeutic option in moderate to severe acne vulgaris.

\section{References}

[1] Suh, D. H et al. A Multicenter Epidemiological Study Of Acne Vulgaris In Korea. International Society of Dermatology. 2011 50: 673-81.

[2] Anwar A I. Procedures for Acne Vulgaris. Makassar. Two One Pree. 2013.

[3] Seth V \& Mishra A. Acne Vulgaris Management; what's new and what's still true?. Int J Adv Med. 2015: 2 (1): 1-5.

[4] Gamble et al. Topical Antimicrobial Treatment of Acne Vulgaris. Am. J Clin Dermatol. 2012. 13: 141-62.

[5] Hanafi M J. Haid dan Siklusnya, In: Wiknjosastro, H., Saifuddin, AB \& Rachimhadhi, T. (Eds.) Science Content Tridasa Printer, Jakarta: YayasanBima. Pustaka Sarwono Prawirohardji 2007.

[6] Elsaie M L. Hormonal Treatment of Acne Vulgaris; An Update. Clinical, Cosmetic and Investigational Dermatology. 2016. 9: 241-48.

[7] Bakry O A \& Shazly. Role Of Hormones And Blood Lipids In The Pathogenesis Of Acne Vulgaris In Non-Obese, Non-Hirsute Females. Indian Dermatology Online Journal. 2014. 5 (1): 9-16.

[8] Kong Y L \&Tey H L. Treatment of Acne Vulgaris During Pregnancy and Lactation. Drugs. 2013. 73: 779-87.

[9] Meredith F M \& Ormerod A D. The Management of Acne Vulgaris in Pregnancy. Am. J Clin Dermatol. 2013. 14: 351-58.

[10] Fox C \& Gerber. Treatment Modalities for Acne. Molecule. 2016. 21: 1-20.

[11] Aydemir E H. Acne Vulgaris. Turk Ped Ars. 2014. 49: 13-6.

[12] Dawson A L \& Dellavalle R P. Acne Vulgaris. BMJ. 2013. 1-7.

[13] Tahir M. Pathogenesis of Acne Vulgaris; Simplified. Journal of Pakistan Association of Dermatologists. 2010. 20: 93-97. 
[14] Czop B B. The Aetiopathogenesis Of Acne Vulgaris - What's New ?. International Journal of Cosmetic Science. 2014. 36: 187-194.

[15] Malahlela P \& Motswaledi M H. Management of Mild to Moderate Acne Vulgaris. S Afr Fam Pract. 2013. 55 (3): 24144.

[16] Lai K W \& Mercurio M G. Update on the Treatment of Acne Vulgaris. JCOM. 2009. 16 (3): 116-26.

[17] Whitney M \& Ditre C. Management Strategies for Acne Vulgaris. Clinical, Cosmetic and Investigational Dermatology. 2011. 4: 41-53.

[18] Kogawa A C \& Salgado H R N. Doxycycline Hyclate: A Review Of Properties, Applications And Analytical Methods. Pharmaceutical analysis. 2012. 2 (4): 11-25.

[19] Simonart T. Newer Approaches to the Treatment of Acne Vulgaris. Clin Am. J Dermatol. 2012. 13 (6): 357-64.

[20] Werner M. A Systematic Review of the Effects and Side Effects of Treatment with Oral Tetracycline in Acne Vulgaris. Master Thesis in Medicine. 2013.
[21] Davis E C \& Callender. A Review of Acne in Ethnic Skin Pathogenesis, Clinical manifestations, and Management Strategies. Aesthetic J Clin Dermatol. 2010.3 (4): 24-38.

[22] Elahmed H H. Management of Acne Vulgaris with Hormonal Therapies in Adult Female Patients. Dermatology Therapy. 2015. 4: 166-72.

[23] Shu Y Y \& Maibach H I. Estrogen and Skin. Am. J Clin Dermatol. 2011. 12 (5): 297 -311.

[24] Gumanga S K \& Arye R A K. Menstrual Some Characteristics In Adolescent Girls In Accra, Ghana. Ghana Medical Journal. 2012. 46 (1): 3-7.

[25] Edison N et al. Effectivity of Preovulation Phase Autologous Serum Application on Moderate-Severe Acne Vulgaris Patients. American Journal of Clinical and Experimental Medicine. 2016. 4 (6): 170-78.

[26] Ilham. Pengujian tetes serum autolog sebagai antibakteri dan inflamasi pada penyakit permukaan okuler. Makassar, Universitas Hasanuddin. 2011. 